\title{
A Computational Framework to Assess the Efficacy of Cytotoxic Molecules and Vascular Disrupting Agents against Solid Tumours
}

\author{
M. Pons-Salort ${ }^{1}$, B. van der Sanden ${ }^{2}$, A. Juhem ${ }^{3}$, A. Popov ${ }^{3}$, A. Stéphanou ${ }^{1}$ * \\ ${ }^{1}$ UJF-Grenoble 1, CNRS, Laboratory TIMC-IMAG UMR 5525 \\ DyCTiM research team, 38041 Grenoble, France \\ 2 INSERM U836, Grenoble Institut des Neurosciences, UJF-Grenoble 1 \\ CHU Michallon, 38042 Grenoble, France \\ ${ }^{3}$ Ecrins therapeutics, BIOPOLIS, 38700 La Tronche, France
}

\begin{abstract}
A computational framework for testing the effects of cytotoxic molecules, specific to a given phase of the cell cycle, and vascular disrupting agents (VDAs) is presented. The model is based on a cellular automaton to describe tumour cell states transitions from proliferation to death. It is coupled with a model describing the tumour vasculature and its adaptation to the blood rheological constraints when alterations are induced by VDAs treatment. Several therapeutic protocols in two structurally different vascular networks were tested by varying the duration of cytotoxic drug perfusion and the periodicity of treatment cycles. The impact of VDAs were also tested both experimentally from intravital microscopy through a dorsal skinfold chamber on a mouse and numerically. Simulation results show that combining cytotoxic treatment with a post treatment of VDA through a judicious timing could favour the rapid eradication of the tumour. The computational framework thus gives some insights into the outcome of cytotoxic and VDAs treatments on a qualitative basis. Future validation from our experimental setup could open up new perspectives towards Computer-Assisted Therapeutic Strategies.
\end{abstract}

Key words: computational modelling, cellular automaton, cytotoxic molecules, vascular disrupting agents, vascular tumour growth, therapeutic protocols

\footnotetext{
*Corresponding author. E-mail: Angelique.Stephanou@imag.fr
} 
AMS subject classification: 92-08, 92B05, 92C15, 92C37, 92C42, 92C50, 92D15

\section{Introduction}

Cancer is one of the major causes of death in the world. The complex and interrelated pathological processes underlying its development have attracted the attention of scientists with different backgrounds. Research dedicated to understand and fight cancer is consequently one of the most active areas in biomedicine.

One of the most common therapies to treat solid tumours is chemotherapy with the use of cytotoxic drugs. These molecules aim at killing tumour cells by stopping or preventing cell proliferation. More recently, antivascular treatments started to be used and many are in clinical trials. Among the antivascular treatments, we can cite the antiangiogenic therapy which prevents the vascular endothelial growth factors (VEGF), produced by the tumour cells to promote vascular growth, from binding with receptors at the surface of endothelial cells. The consequence of this therapy is to limit the pathologic formation of new blood vessels [7, 10]. This idea to act on the tumour vasculature rather than on the tumour itself was proposed for the first time in 1971 by $\mathrm{J}$. Folkman [11]. The principle was to cut the tumour from its blood supply in order to kill the cancer cells through starvation or alternatively to prevent the tumour from growing to a more harmful state by keeping it dormant. Thirty years later, in 2001, R.K. Jain proposed that this form of therapy, judiciously applied, could also be used to "normalize" the vasculature, that is to prune most of the smallest abnormal vessels while keeping its backbone, in order to improve the delivery of therapeutic molecules to the tumour [18]. A more drastic case in antivascular treatment is the use of vascular disrupting agents (VDAs) which directly destroy the unmature tumour vasculature. This treatment acts almost instantaneously after its injection to stop the tumour perfusion leading to widespread tumour necrosis [45]. However the most peripheric tumour cells survive and induce tumour regrowth. This is why VDAs should preferentially be used in combination with other therapies [38].

Today the big challenge, in order to significantly improve cancer therapy for the many different types of solid tumours, is to be able to couple efficiently the existing different means of action. The combinations of therapies are extensively explored theoretically [40,30, 9, 48, 28] often ahead of experiments. The aim for therapeutic combinations is first, to enhance the curing potential and second to limit toxicity since smaller doses of each therapeutic molecules can be used. One classic example of therapeutic combination is the use of antivascular molecules, which target the vascular network, with molecules which directly targets the cancer cells (proliferating cells) as proposed by R.K. Jain [18]. Indeed, depending on the properties of the vasculature (density, connectivity, maturity) antivascular drugs can either disrupt the vascular network and stop the delivery of cytotoxic molecules or can create the opposite effect by normalizing the vasculature $[40,9]$. In that latter 
case, blood access to the tumour is improved, which means a better drug delivery to kill cancer cells, but at the same time an increased nutrients and oxygen delivery favouring their growth. For example, enhanced effects of chemotherapeutic agents and radiotherapy have been reported when used in combination with antivascular agents $[36,37]$. Novel therapeutic strategies consist in the optimization of dosage and schedule for these combined therapies for individual patients $[7,18]$. They have proven to be very successfull for a number of cancer except for gliomas where some recent studies have shown that antiangiogenic treatment induces increased local invasion and distant metastasis [29].

The development of theoretical models, which can both reflect pathological cases and predict the outcome of treatments, has a significant impact in this therapeutic area [43, 34, 44, 48]. Mathematical and computational models are extensively developed in that sense. Such models can be used to determine the most efficient combinations of therapies, the delivery regimes (optimum schedule, site of delivery, etc.) with the most effective being taken further in the clinical settings [16]. This was made possible thanks to the tremendous improvements of the tumour growth models over the past ten years $[46,6]$. The current models are able to describe the tumour evolution from avascular to vascular growth. They integrate blood flow and vascular adaptation due to rheological constraints [1, 41, 23, 24]. They integrate vascular remodelling in connection with the tumour mass evolution and allow to take into account the effects of treatment [14]. Models are now trully multiscale from the molecule to the tissue around the central cell scale [4, 21, 20, 35, 31]. Intracellular signalling cascades and their implication at the organ scale can now be apprehended and reciprocally. To achieve this, hybrid modelling was developed to access and to be able to describe the full complexity of the multiple events at stake in the tumour evolution [6,27]. These models are based on the coupling between different theoretical formulations, such as a computationally based cellular automaton with a mathematically based system of partial differential equations. Automata are often used at the smallest scale and allows one to deal with the interactions between individual entities (molecules, cells, etc.)

In this paper, we propose to use such an integrated model of the tumour evolution to specifically test two different therapeutic molecules, a cytotoxic molecule specific for a particular phase of the cell cycle and an antivascular agent, more specifically a vascular disrupting agent (VDA). The mode of action differs from antiangiogenic agents since VDAs act by disrutping the tumour vasculature rather than preventing its growth [45]. It can thus be used at a more advanced stage in the tumour development. We present a computational model specifically designed to evaluate the impact of these drugs and their potential combination. Our aim was to identify the main scenarios, on a qualitative basis, that may occur by using these particular drugs.

In a first part we compare the effects of two phase-specific cytotoxics, acting either on long or short phases of the cell cycle. The molecules are perfused in two structurally different vascular networks according to three different protocols which differ by varying the duration of the drug perfusion and the periodicity of the perfusion cycles. Consequences for the setup of effective therapies are drawn from the results. In a second part, the effects of the VDAs are illustrated 
on a mouse bearing a tumour in a dorsal skinfold chamber, using intravital microscopy. These effects are then simulated from the computational model. Combination with cytotoxic molecules are finally considered and enlighten the condition for judisciously using VDAs.

\section{The computational model}

The computational model aims at providing a framework for testing two types of therapeutic molecules: cytotoxic molecules directed against the tumour cells and VDAs directed against the vascular network.

Cytotoxic molecules - These molecules generally act on the microtubules to block the process of cell division. They are mainly phase-specific, which means that the cells are only sensitive to these molecules while in a particular phase of the cell cycle. To evaluate the impact of these molecules on tumour growth, it is required to take into account the cell dynamics by which the cell can change state depending on the environmental conditions. Those are limited in this model to the amount of oxygen, the available space and the drug concentration.

$V D A s$ - These agents specifically target the endothelial cells of the existing tumour vasculature through several pathways and cause a pronounced shutdown in blood flow to the tumour tissue, while leaving the blood flow in normal tissues relatively intact $[19,45]$. The collapse in tumour blood flow is detected within minutes [45]. Vascular disruption leads to extensive zones of hypoxia which results in tumour cells necrosis within days.

The diagram in figure 1 summarizes the relationship between the elements and processes integrated in the computational model. Those are detailed in turn in the following sections.

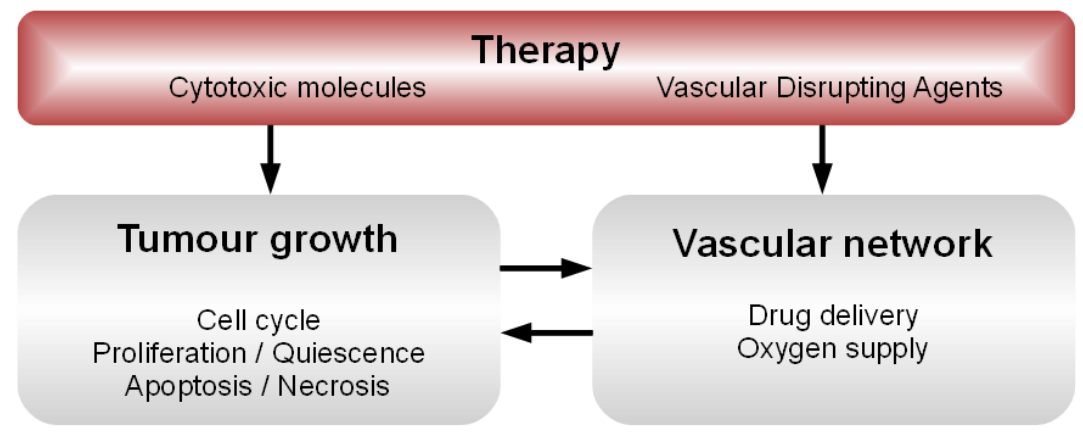

Figure 1: Relationship between the elements of the computational framework. 


\subsection{Tumour growth}

\subsubsection{Cell cycle}

The cell division cycle is the sequence of events by which a cell replicates all its components and divides them into two daughter cells. It is usually divided into four phases: $G_{1}, S, G_{2}$ and $M$ [47]. The cell-cycle can be affected by the lack of extracellular oxygen (hypoxia), but the effects are different in normal and cancer cells. Both cell types experience hypoxia-induced arrest of the $G_{1}$ phase of the cell-cycle, but only cancer cells become quiescent by entering the $G_{0}$ phase (Fig. 2 ). This corresponds to the transition of the cell to a latent state in which most of its functions, including proliferation, are suspended. Quiescent cells can survive under hypoxia for long periods and they can come back to the proliferative state when more oxygen becomes available. This ability of cancer cells to go into a quiescent (dormant) state gives them a higher resistance to hypoxia, although if the level of oxygen becomes extremely low or hypoxia persists for a long time, cancer cells eventually die [2, 47, 3]. Alternatively, cancer cells can also change their metabolism to glycolysis to cope with the deprivation of oxygen. There is a lack of knowledge about how long a quiescent cell can survive in $G_{0}$ phase. We make the assumption that if a cell stays more than 10 days in hypoxic conditions, it will die from necrosis. It is known that the cell doubling time varies from one tissue to another, and also between cells of the same tissue (in this case, the difference lies in the variations of the $G_{1}$ phase duration). Durations for the cell cycle reported in glioma range from 24 to 72 hours $[17,32,25,39]$. In this model, it is assumed that each cell has the same cell-cycle duration of about 3 days (from 69 to 72 hours).

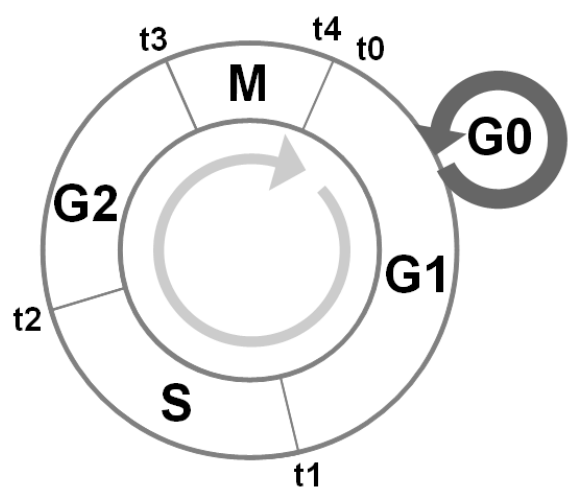

Figure 2: Graphical representation of the cell-cycle. Cells in the $G_{1}$ phase experiencing hypoxia enter the $G_{0}$ phase which marks the transition to quiescence. They can come back to the $G_{1}$ phase as soon as the level of oxygen becomes sufficient again. We assume here that the cell cycle lasts about 3 days (from 69 to 72 hours) and the following durations for each phase are taken: $G_{1}$, $t_{1}-t_{0}=30 h ; S, t_{2}-t_{1}=12 h ; G_{2}, t_{3}-t_{2}=27 h$ and $M, t_{4}-t_{3}=3 h$. 


\subsubsection{Cell metabolism}

All cells consume nutrients and oxygen to survive and accomplish their tasks. Cancer cells consume oxygen at rate $2.3 \times 10^{-16} \mathrm{~mol} \mathrm{cell} \mathrm{s}^{-1} \mathrm{~s}^{-1}$ [13]. The base consumption rate of proliferative cells is consequently set to this value that we denote $\alpha_{p}$. The fact that quiescent cells consume less oxygen than active ones is introduced by dividing their consumption rate by a factor of 5 [8]. A proliferative cell becomes quiescent if the local oxygen concentration is lower than $\alpha_{p}$. Inversely, if the local oxygen concentration of a quiescent cell increases up to $\alpha_{p}$, it comes back to the proliferative state. If oxygen level drops below $\alpha_{q}=\alpha_{p} / 5$ [12] and if the cell is exposed for too long to such extreme condition, it dies of hypoxia and becomes necrotic. In this model, nutrients are not taken into account, and oxygen is the only substance responsible for cell growth. We note that some tumour cells are able to switch to an anaerobic energy metabolism based on the glycolysis. However, we will not consider this case in our model at this stage.

\subsubsection{Cell death}

Cancer cell death can occur through two different mechanisms: apoptosis or necrosis. Proliferating cells die through apoptosis if they are exposed to a high concentration of cytotoxic molecules. Quiescent cells in the other hand are resistant to these molecules, but they can die through necrosis if the level of oxygen becomes extremely low or if hypoxia persists for a long time (that is beyond a time limit that we set to $T_{\text {limit }}=10$ days). In case of apoptotic death (programmed cell death) the dead cell fragments are cleaned up by macrophages in a few hours (we set this to $t_{\text {apop }}=5$ hours), whereas in necrotic death the dead cell debris remain and form necrotic aggregates.

\subsubsection{Cell state transitions}

The tumour cells evolution is modelled by a cellular automaton inspired from the one previously described in [1]. It is defined on a $G$ grid (Fig. 3) where each element $e$ can be empty $(e=0)$ or contain a tumour cell which can either be proliferative, quiescent, apoptotic or necrotic $(e=1,2,3$ or 4 respectively).

The transitions between the different cell types are conditioned by the cell cycle and environmental conditions. More specifically, the level of oxygen available in the tissue, the available space and the local drug concentration determine if the cell will proliferate or die. A timer $t_{P}$ is assigned to each new born proliferative cell and initially set to $t_{P}=0$. The timer is incremented in the simulations with time step $\Delta t$ to follow the cell progression in its cycle. The rules for state transition of the tumour cells described in the flowchart (Fig. 4) are as follows:

- Only proliferative cells are sensitive to cytotoxic drugs. If the local concentration of drug is lethal the cell dies if it is in the related sensitive $X$ phase for this drug (in this paper, $X=G 2 M$ or $S$ ). If the cell is not in the sensitive phase, it survives if there is enough oxygen and space.

- A proliferative cell becomes quiescent if the level of oxygen is below the admissible threshold for proliferation $\alpha_{p}$, the cell should additionaly be phase-sensitive to hypoxia, that is in 


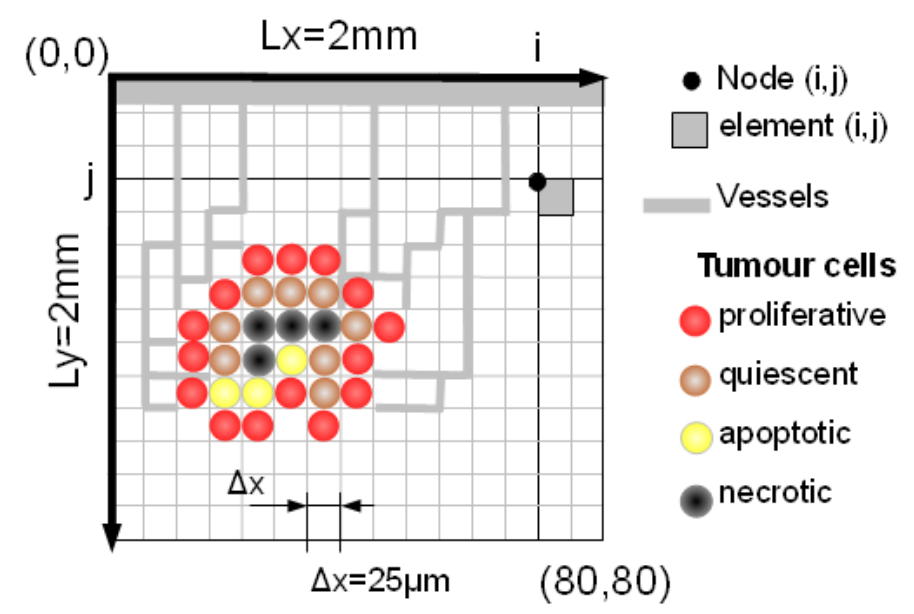

Figure 3: The simulation domain is a $2 m m$-long square piece of tissue $\left(L_{x}=L_{y}\right)$, discretized by a regular $(i, j)$-grid $G$ with $80 \times 80$ elements. The resulting size of each element $e$ is $\Delta_{x}=\Delta_{y}=$ $25 \mu \mathrm{m}$ so as to correspond to the approximate size of a real cell. A tumour cell thus occupies an element $e(i, j)$ of the grid and a vessel segment is defined between two horizontal or vertical nodes $N(i, j)$ of this grid.

G1 phase .

- If the level of oxygen is above the admissible threshold $\alpha_{p}$, then the cell can divide if in $M$ phase provided there is enough available space. This means that an empty element $e=0$ adjacent to the cell must exist to receive one of the daughter cell. The other daughter cell replaces the mother cell.

- Tumour cells are not able to regulate their proliferation. Therefore, if there is not enough space then the initial proliferative cell enters apoptosis. The apoptotic stage lasts for a time $t_{\text {apop }}$ during which the cell keeps occupying the grid element until the cell is removed which corresponds to the release of this grid element.

- A quiescent cell can come back to a proliferative state if the level of oxygen becomes normal again $\left(>\alpha_{p}\right)$. However, if the oxygen level keeps decreasing below the admissible quiescence threshold $\alpha_{q}$ and if the cell is exposed for too long to this increased stress (for longer than time $T_{\text {limit }}$ ) then it becomes necrotic. In this case, the grid element occupied by the cell is never released. 


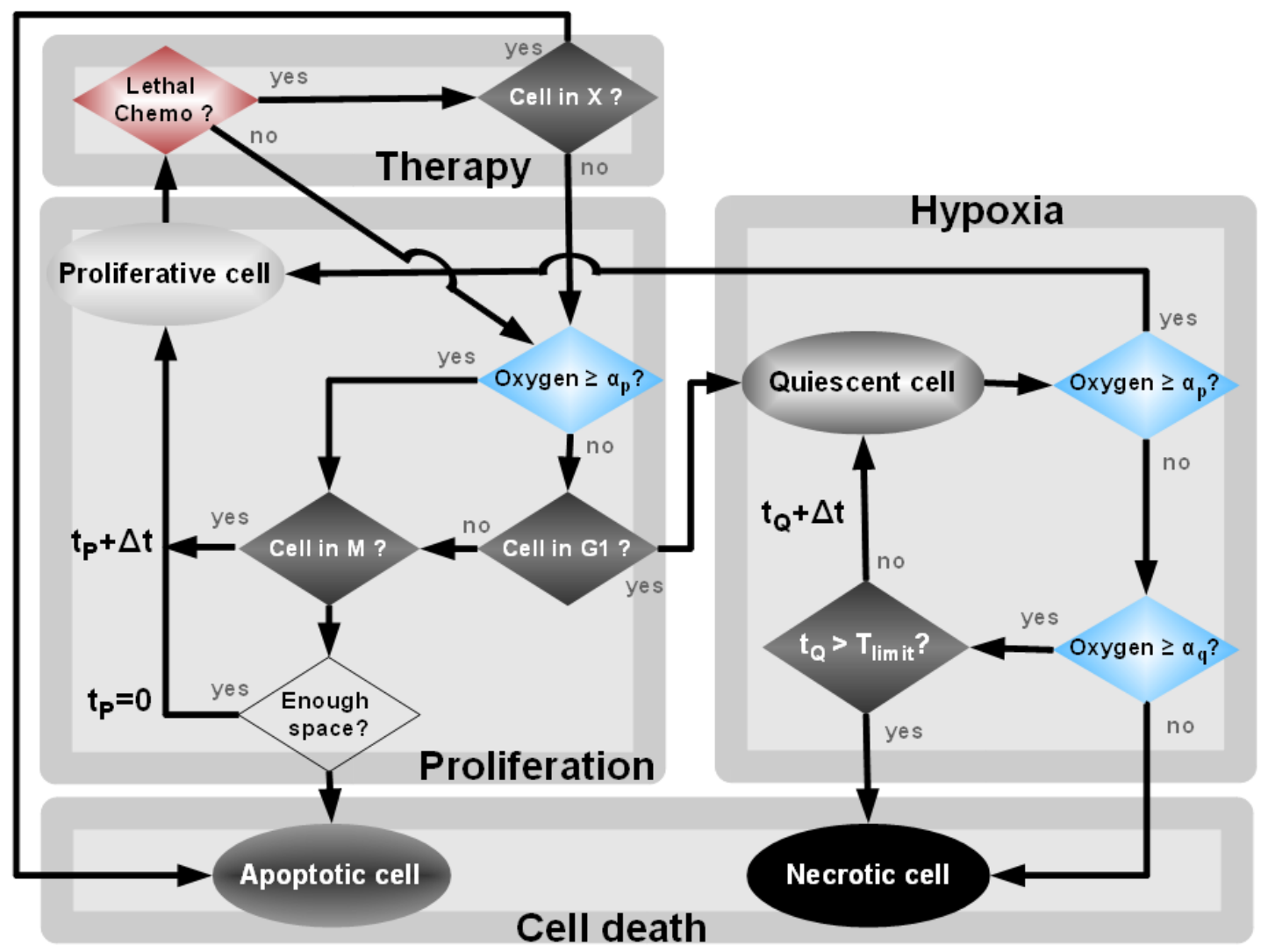

Figure 4: Flowchart for tumour cell states transitions. A timer $t_{P}=0$ is assigned to each new proliferative cell. In normal oxygen conditions the timer is incremented with time step $\Delta t$. When a cell enters hypoxia, that is the $G_{0}$ phase, its timer $t_{P}$ is stopped and switched to $t_{Q}=0$. As long as the cell remains quiescent, its timer $t_{Q}$ is incremented by $\Delta t$. If oxygen becomes available again, the cell returns to the proliferation state and its timer is switched back to its value $t_{P}$ at which it was stopped. If $t_{Q}>T_{\text {limit }}$ then the cell becomes necrotic and switches irreversibly to timer $t_{N}$ which runs to infinity. The cell can also die by apoptosis (programmed cell death). It happens in this automaton if space is lacking for cell division. In that case, the cell timer is also switched irreversibly to $t_{A}$. It is incremented by $\Delta t$ until it reaches its limit $t_{A}=t_{\text {apop }}$ above which the cell is killed and cleaned from the simulation space.

\subsection{Vascular network}

\subsubsection{Angiogenesis and blood flow}

In this paper, the vascular networks that perfuse the tumour are generated from the tumour angiogenesis model described in [41] where all details can be found. The growth of the vasculature results from the migration of endothelial cells detaching from a parent vessel, in response to the combination of chemotactic and haptotactic gradients of tumour angiogenic factors $(c)$ and matrix fibres $(f)$ respectively. The migration of the endothelial cells is expressed by equation (2.1) which 
reflects the probability for a cell $n$ to move in one particular direction of the $2 \mathrm{D}$ space discretized using the coordinates $(i, j)$ as in the grid of figure 3 .

$$
n_{i, j}^{t+1}=P_{0} n_{i, j}^{t}+P_{1} n_{i+1, j}^{t}+P_{2} n_{i-1, j}^{t}+P_{3} n_{i, j+1}^{t}+P_{4} n_{i-1, j}^{t}
$$

Each coefficient, $P_{k}, k=0$ to 4 , represents the probability for the cell to remain stationary $\left(P_{0}\right)$, to move left $\left(P_{1}\right)$, right $\left(P_{2}\right)$, up $\left(P_{3}\right)$ or down $\left(P_{4}\right)$. These probabilities depend on the local gradients of tumour angiogenic factors $(\nabla c)$ and matrix fibres $(\nabla f)$, that is:

$$
P_{k}=P_{k}(D, \chi \nabla c, \rho \nabla f),
$$

where the coefficient $D$ is a diffusion coefficient accounting for the random displacement of the cell. The coefficients $\chi$ and $\rho$ regulate the sensitivity of the endothelial cells to the chemotactic and haptotactic gradients respectively. Adaptation of these coefficients allow one to control the structure of the resulting vascular network in terms of density and connectivity. The probability for the growing vessels to form some branches is assumed to be proportional to the local density of angiogenic factor.

Vascular adaptation due to blood flow is taken into account in this model. Hemodynamic and metabolic stimuli either dilate or constrict the vessels and ultimately lead to a realistic vascular network typical of the tumour vasculatures (Fig. 5). We note that this model of vascular tumour growth and adaptation to rheological constraints was subsequently developed in the following contributions [23, 21, 24].

Two vascular networks are considered in the simulations to perfuse the tumour. A first relatively homogeneous network (Fig. 5A) and a second more heterogeneous network with higher vessels density (Fig. 5B) obtained by increasing the endothelial cells sensitivity to the tumour angiogenic factors from the mathematical model described in [41]. The tumour at this stage is not explicitely materialized. It is only represented by a VEGF gradient increasing from the top to the bottom of the simulation frame.

In a second step, a perfectly circular tumour (half disk) with all tumour cells initially proliferative, is superimposed on those vasculatures (Fig. 5). Therefore, the imposed tumour geometry plays no explicit part in the generation of the vascular networks used. This means that the initial conditon is "artificial". However the tumour spontaneously adapts to the imposed vasculatures (during the first few iterations of the simulations), since the unperfused tumour regions quickly die.

\subsubsection{Oxygen diffusion}

Oxygen is transported by the red blood cells, which proportion in the blood volume is given by the hematocrit. Oxygen molecules diffuse from the vessels to the tissue. In this model, the amount of oxygen molecules is therefore assumed to be directly proportional to the blood hematocrit. The equation describing the oxygen concentration in each automaton element $e$ is given by: 


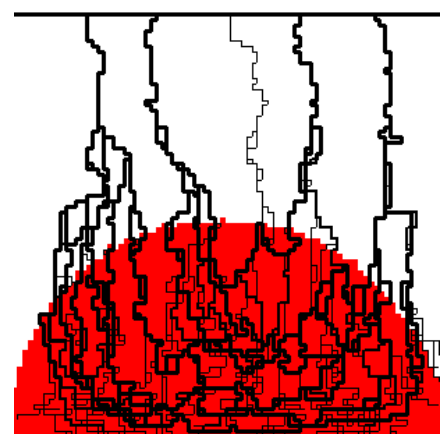

(A) Network 1

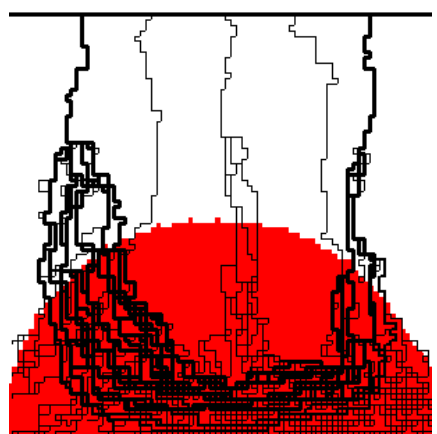

(B) Network 2

Figure 5: Vascular networks taken for the simulations to perfuse the tumour. (A) Vascular network 1 perfuses the tumour homogeneously. (B) Vascular network 2 with a higher density of vessels perfuses the tumour heteregeneously leaving a vast area of the tumour unperfused. All the tumour cells are initially proliferative (red).

$$
c_{O_{2}}(e, t)=\sum_{i \in V} \frac{q_{i}}{\sqrt{4 \pi D_{O_{2}} t}} \exp \left(-\frac{x_{i}^{2}}{4 D_{O_{2}} t}\right)
$$

where $V$ is the set of vessels located at a distance $x_{i}$ smaller than $0.1 \mathrm{~mm}$ from the automaton element $e$ and $q_{i}$ is the oxygen quantity in the vessel $i$ evaluated from the hematocrit $[1,41] . D_{O_{2}}$ is the oxygen diffusion coefficient with value $D_{O_{2}}=1.8 \times 10^{3} \mu \mathrm{m}^{2} / \mathrm{s}$ [15].

\subsection{Therapies}

\subsubsection{Cytotoxic molecules}

Cytotoxic molecules block the process of cell division and therefore only affect proliferative cells. Most of these molecules are phase specific meaning that only cells in the appropriate phase are sensitive to treatment. In this model we propose to test two molecules: one affecting the long G2M phase and another one affecting the shorter $\mathrm{S}$ phase. The most common method of administration is the intravenous one, which allows the drug a rapid entry in the body circulation. Doses can be given as a bolus or can be perfused over a few hours or days. In general the treatment is given in cycles. In our model, the molecules are perfused in the host vessel of the vascular neworks. Perfusions last from 5 to 10 hours and are performed in cycles of one or two weeks. Diffusion of the cytotoxic molecules is described with the same equation as eq. (2.3) replacing $D_{\mathrm{O}_{2}}$ with $D_{\text {cyto }}=0.5 \times 10^{3} \mu \mathrm{m}^{2} / \mathrm{s}[42,26]$ and taking $0.05 \mathrm{~mm}$ as the maximum diffusion length.

\subsubsection{Vascular disrupting agents}

VDAs operate by disrupting specifically the endothelium of solid tumours resulting in the death of tumour cells from lack of oxygen and nutrients. This leads to the occlusion of blood-transporting vessels as well as the capillary sprouts, resulting in widespread necrosis of established tumours 
[19]. A rapid collapse in tumour blood flow can be completed and detected within minutes and is maintained for over 60 minutes after initial vascular shutdown [45]. In some clinical trials, vascular parameters returned to baseline values by 24 hours after treatment [45]. In our model, we assume that VDAs disrupt the vessels perfused with a high concentration of drug and bearing a low shear stress for allowing the drug to act on the endothelium. Vessels disruption then leads to vascular readaption due to the re-circulation of the blood flow through the altered vascular network. Details on the modelling of this adaptation effect can be found in [41]. VDAs are administered through a 5 second bolus injection into the host vessel of the vascular networks.

\section{Results}

\section{Initial condition and model limitations}

The initial condition corresponds to an advanced stage of cancer where the tumour is already vascularized. Since our aim is to test the impact of therapies, we chose an idealized (hence artificial) case where the tumour is initially semi-circular and superimposed on the vasculature (Fig. 5). All tumour cells are initially set as proliferative and are randomly assigned an age in the cell cycle.

We note that one limitation of the model is the partial coupling considered between the vascular network and the tumour. Those two are grown independently: the vasculature is obtained from a VEGF gradient (see section 2.2.1.) and the tumour geometry is imposed as a half-disk. In reality the vascular network develops in response to VEGF signals produced by the tumour cells and reciprocally the tumour develops depending on the nutrients brought by the vasculature. The growth of the vasculature and tumour are thus tightly interdependent. In this model, we do not consider the feedbacks from the tumour on vascular growth or degradation since the vascular network is unaltered in our simulations. However, we do consider vascular disruption due to VDAs. The subsequent vessels adaptation, due to the new blood flow conditions in the altered vasculature, is also taken into account. But vascular regrowth (angiogenesis) is not.

The results presented here aim at illustrating that from a relatively simple model which incorporates some basic elements of vascular-tumour interactions, it is possible to tackle on a qualitative basis the issue of therapy. Model refinements can later be addressed.

\subsection{Tumour growth without therapy}

Figure 6 presents the growth of the tumour perfused with the vascular network 1 when no therapy is applied. Snapshots of the tumour are taken every 3 or 4 weeks and show a steady growth rate over 18 weeks. The tumour mass grows mainly along the feeding vessels where the oxgen concentration is high and favours cell proliferation. Apoptotic death due to the lack of space for cell proliferation in the most inner regions of the tumour allows the cells to be renewed and to keep an active pool of proliferating cells. We note that in this current version of the model, the tumour vasculature 
is unaltered by the growing tumour which means that the oxygen concentration remains sufficient for cells renewal. Isolated patches of necrotic cells rapidly appear in the tumour regions the most distant from a feeding vessel and stabilize in a few days. These necrotic occurences are essentially due to the "artificial" initial condition that we impose, since we superimpose a circular tumour to an existing vasculature instead of growing them simultaneously.

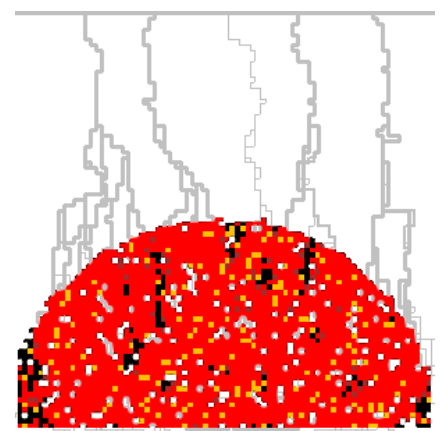

$t=13$ hours

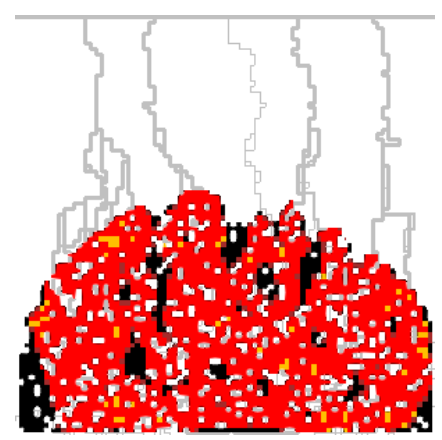

$t=3$ weeks

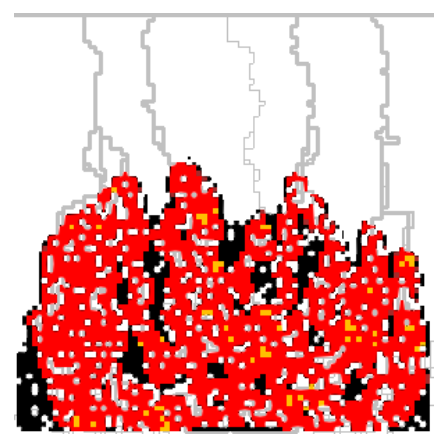

$t=6$ weeks

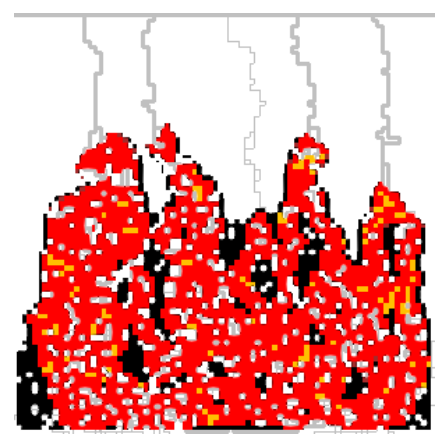

$t=10$ weeks

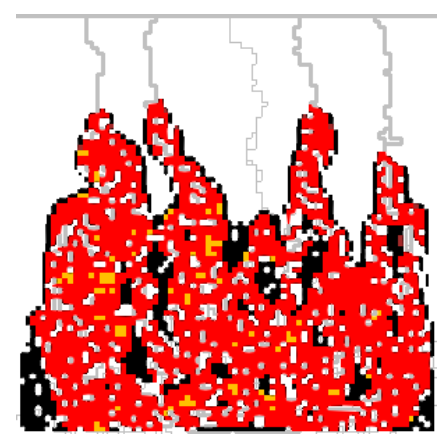

$t=14$ weeks

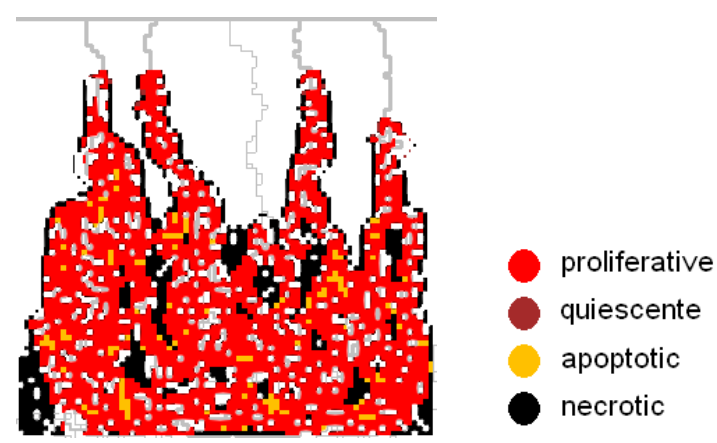

$t=18$ weeks

Figure 6: Tumour growth without therapy. Fingering growth along the feeding vessels is observed over several weeks. The colour legend for tumour cells is the same as in figure 3. 


\subsection{Cytotoxic molecules}

The effect of phase-specific cytotoxic molecules are simulated for the two vascular networks. We define three protocols inspired from those existing in clinical trials [5] with the aim of assessing the effects of two parameters: the amount of drug delivered that we assume directly related to the duration of the drug perfusion and the frequency of the perfusion cycle. The protocols used are as follows:

- protocol A: 5 hours of perfusion once a week,

- protocol B: 10 hours of perfusion once a week,

- protocol C: 10 hours of perfusion once every two weeks.

Figure 7 presents the evolution of the proliferative cell population for each simulated case. For each protocol and each vascular network, two cytotoxic molecules are tested: one targets the 30 hours long G2M phases and the other the shorter 12 hours long S phase. In all cases, it is obviously the molecule targetting the longest part of the cell cycle that is the most efficient since the probability for the tumour cells to be in the sensitive phase is much higher. The tumour is indeed eliminated in 3 to 4 cycles of cytotoxic perfusions whereas it cannot be eradicated with the drug targetting the short $\mathrm{S}$ phase. In that case, we observe that the amount of cells killed after each perfusion session corresponds to the amount that regrows during one period of the therapy cycle. The amount of tumour cells is under control as long as the therapy is maintained, however if it is interrupted, the tumour will regrow.

We note that the results obtained for the two vascular networks are qualitatively the same. Indeed, the unperfused tumour regions in vascular network 2 cannot be reached by the cytotoxic molecules, but they cannot be reached by the oxygen either. This means that the cells located in these unperfused regions die of hypoxia. We thus have a natural adaptation of the tumour to the imposed vasculature which attenuates the crudeness of such initial conditions. The higher vessels density of network 2 in the surviving part of the tumour however contributes to a more important decrease of the amount of proliferative cells with an average decrease of $75 \%$ for the first injection of G2M-cytotoxic molecules, compared to an average $65 \%$ for the same molecules in network 1 .

Figures 8, 9 and 10 show the tumour evolution in three different therapeutic cases. Figure 8 shows the effect of a G2M-cytotoxic molecule perfused according to protocol $\mathrm{A}$ in a tumour vascularised with network 1 (this corresponds to the black curve in figure 7A). The amount of proliferative cells decreases massively in 3 weeks and falls below the detection threshold (set at $2 \%$ of the initial amount of tumour cells). In the other hand, if the S-cytotoxic molecules is used in the same conditions, regeneration of the proliferative cell population is observed in figure 9 (this corresponds to the grey curve in figure 7A). Figure 10 shows the action of the G2M-cytotoxic molecule perfused according to protocol $\mathrm{C}$ in network 1 (corresponding to the black curve in figure $7 \mathrm{E})$. In that case, it takes longer (10 weeks) for the proliferative cell population to decrease below the detection threshold. 


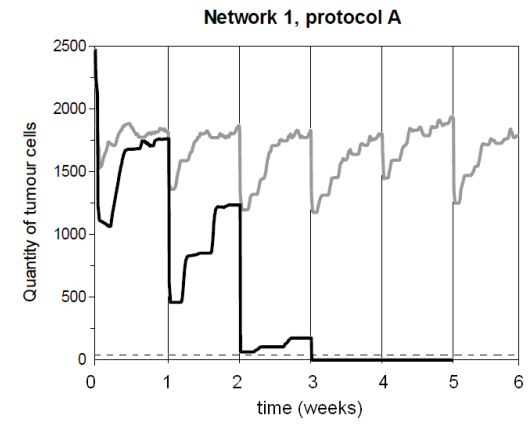

(A)

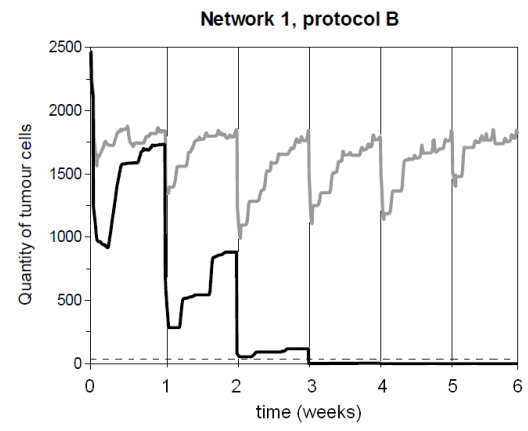

(C)

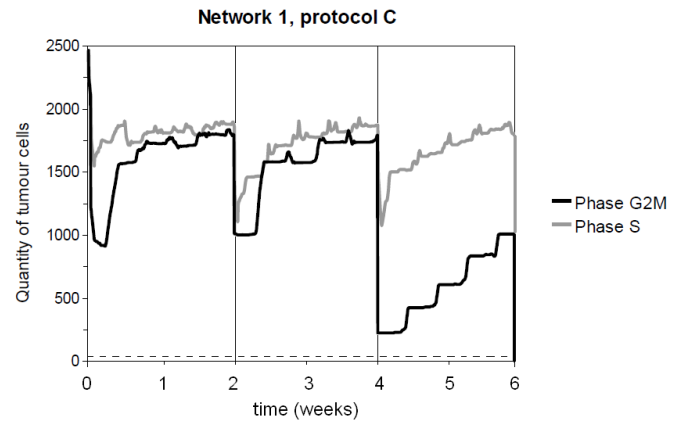

(E)

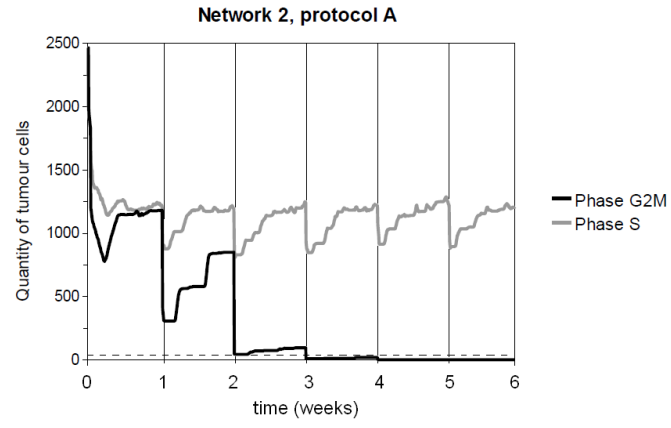

(B)

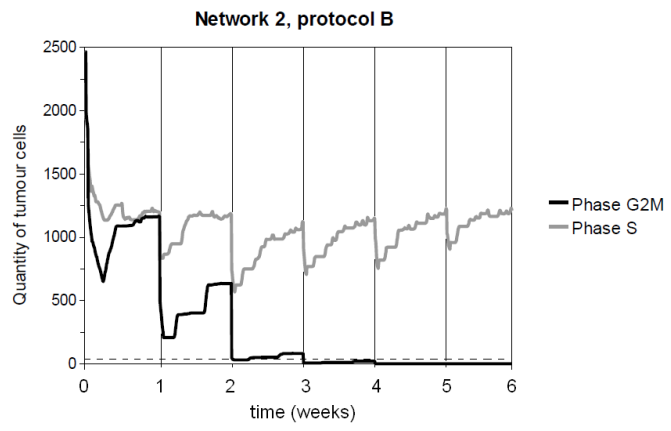

(D)

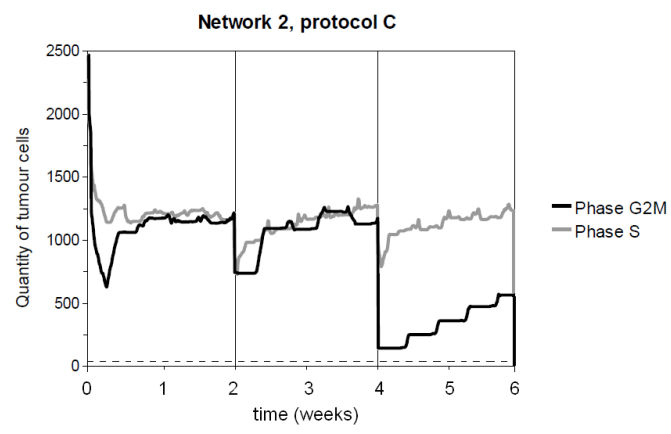

(F)

Figure 7: Evolution of the proliferative cell population depending on the vascular network perfusing the tumour: $(\mathrm{A}, \mathrm{C}, \mathrm{E})$ for network 1 and $(\mathrm{B}, \mathrm{D}, \mathrm{F})$ for network 2 ; the phase of the cell cycle which is targetted by the cytotoxic molecules ( $\mathrm{G} 2 \mathrm{M}$ or $\mathrm{S})$; the therapeutic protocol used: protocol A (A,B), protocol B (C,D) and protocol C $(\mathrm{E}, \mathrm{F})$. The horizontal dashed line corresponds to a detection threshold for tumour cells. 


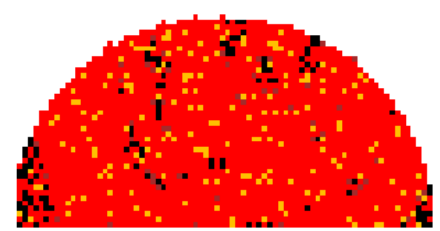

$t=6$ hours

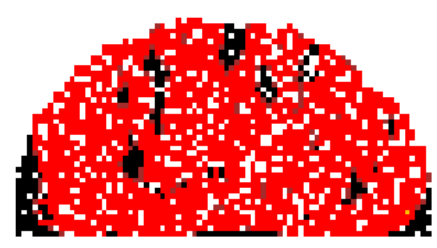

$t=1$ week

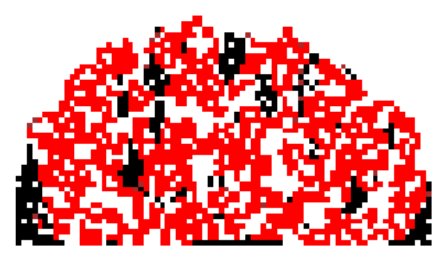

$t=2$ weeks
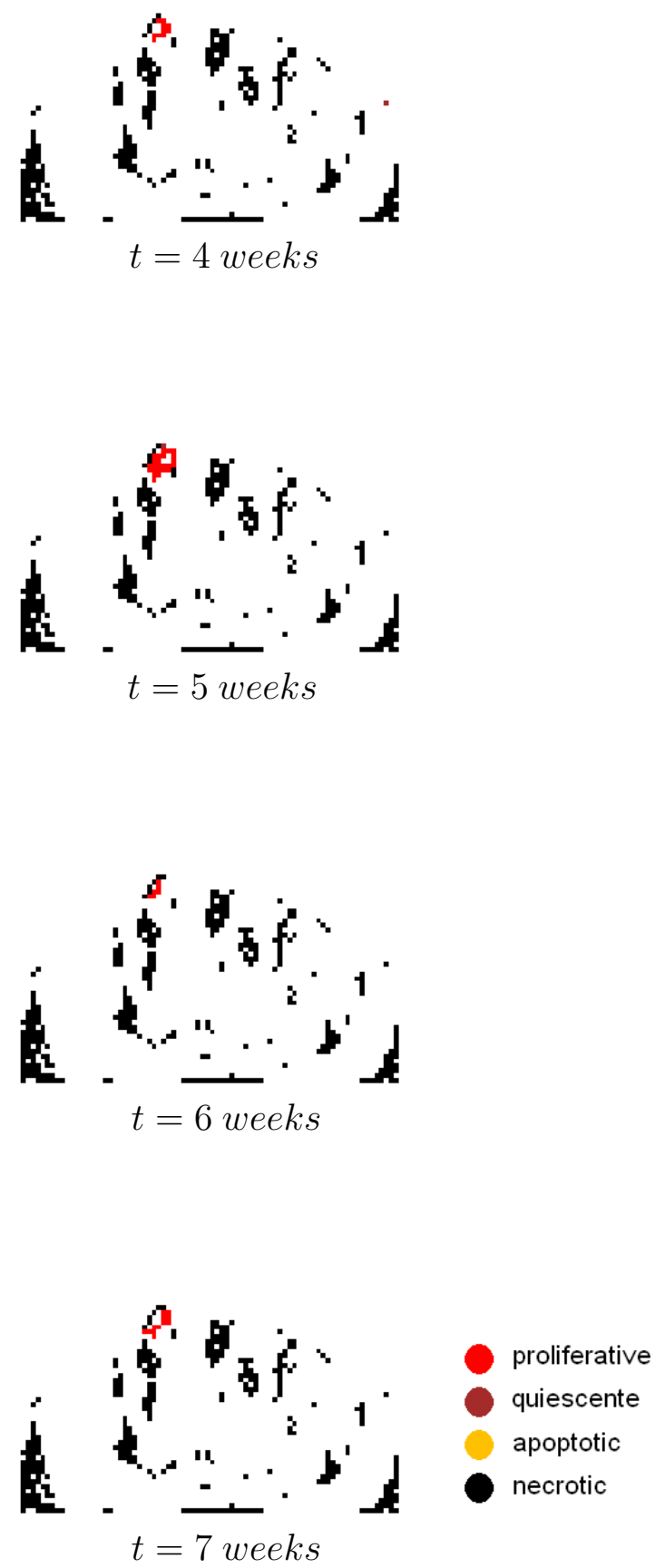

Figure 8: Effects of G2M-phase cytotoxic molecules using protocol A in a tumour perfused with vascular network 1 . 


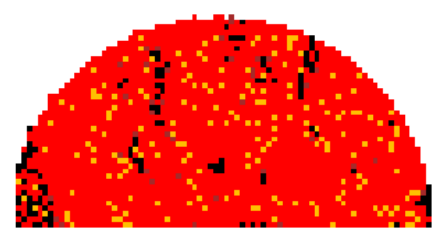

$t=6$ hours

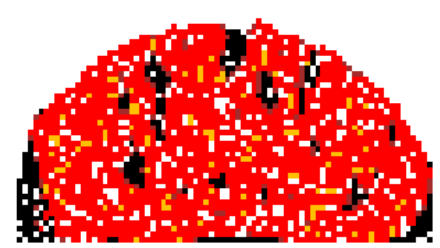

$t=1$ week

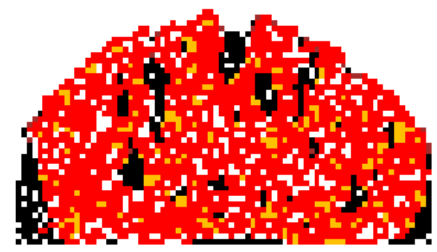

$t=2$ weeks

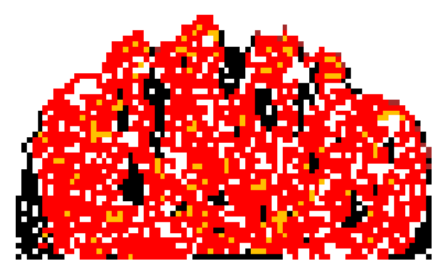

$t=3$ weeks

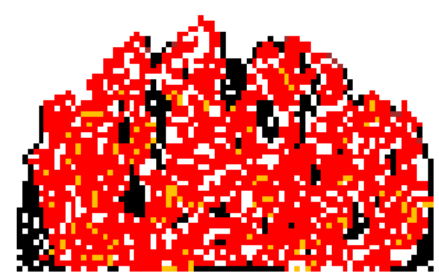

$t=4$ weeks

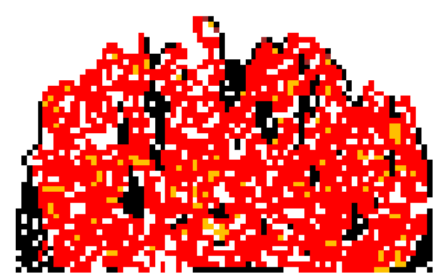

$t=5$ weeks

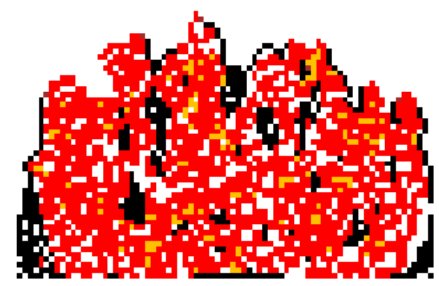

$t=6$ weeks

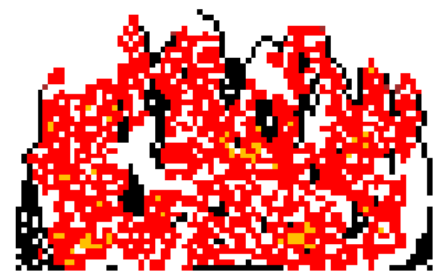

$t=7$ weeks proliferative

quiescente

apoptotic

necrotic

Figure 9: Effects of S-phase cytotoxic molecules using protocol A in a tumour perfused with vascular network 1 . 


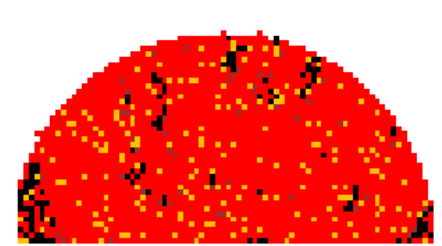

$t=6 h$

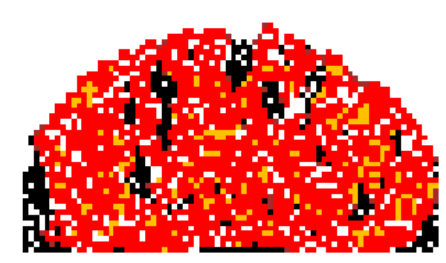

$t=2$ weeks

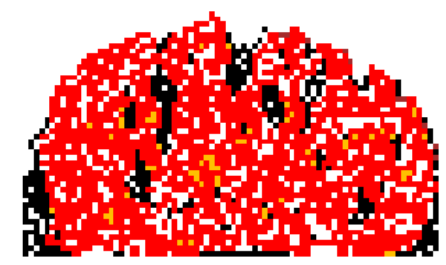

$t=4$ weeks
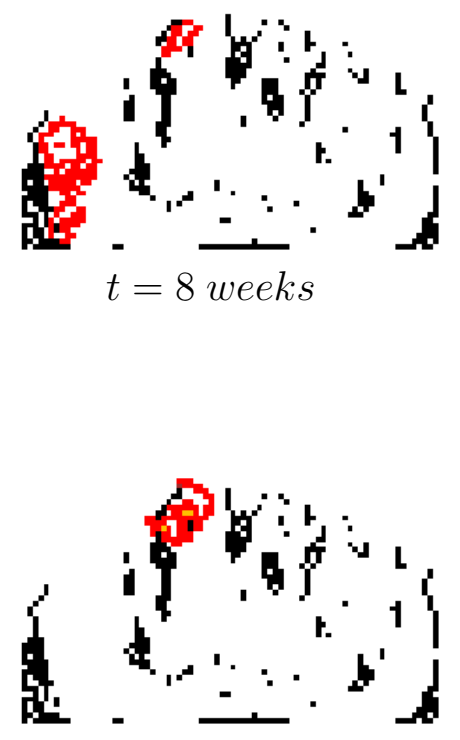

$t=10$ weeks

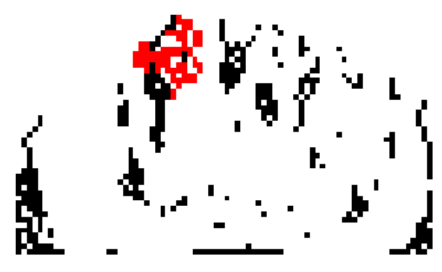

$t=12$ weeks

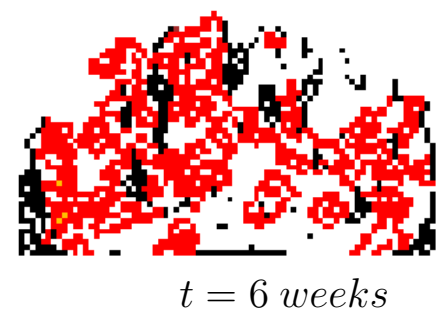

proliferative

quiescente

apoptotic

necrotic

Figure 10: Effects of G2M-phase cytotoxic molecules using protocol $\mathrm{C}$ in a tumour perfused with vascular network 1 . 
Figure 11A compares the three therapeutic protocols used with the G2M-phase cytotoxic molecules perfused in vascular network 1. In all three cases, the amount of proliferative cells eventually falls below the detection threshold. However it takes longer to be reached using protocol $\mathrm{C}$ since the perfusions of cytotoxic molecules are performed with a cycle of two weeks, instead of one for the two other protocols A and B. This gives more time for the tumour to regrow between two cycles. Protocols A and B which differ by the duration of the drug perfusion (twice longer in protocol B than in protocol A, meaning that the amount of drug delivered is twice higher) give identical results. In both cases the tumour is eliminated in 3 cycles of drug administration. Such a result allows us to discard protocol B since doubling the amount of drug does not improve the outcome of the treatment. In the other hand, it might be useful to retain protocol C. Athough the cure takes longer, it could allow the patient more time to recover from potential side effects since drug perfusions are performed on a less frequent basis.

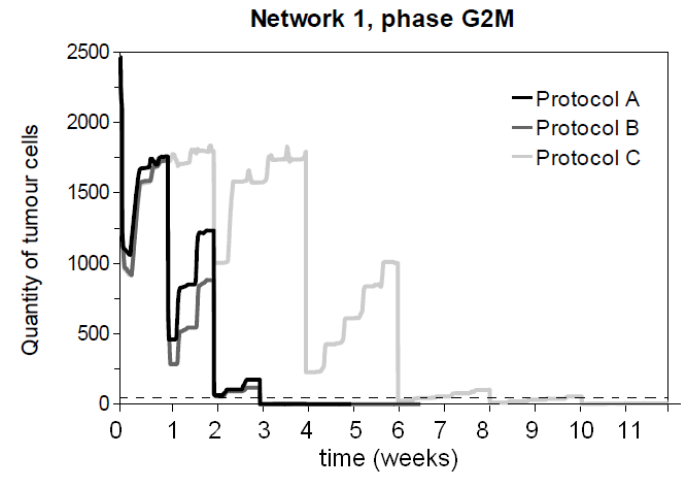

(A)

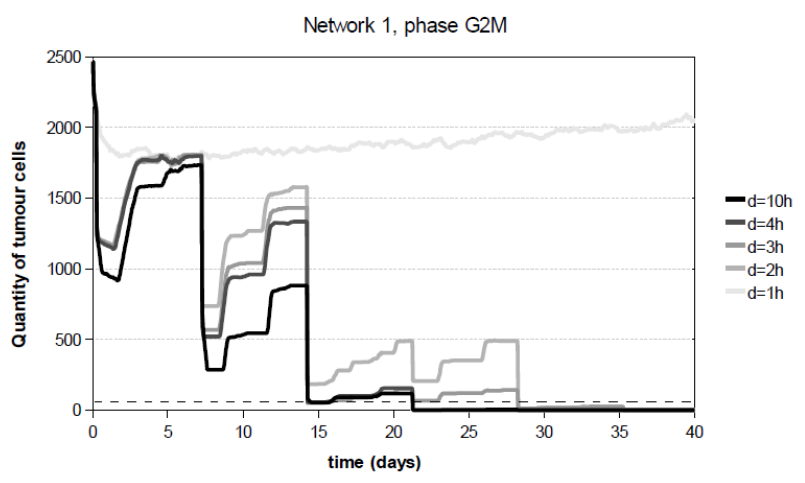

(B)

Figure 11: (A) Comparison of the three therapeutic protocols A, B and C using the G2M-phase cytotoxic molecule perfused in vascular network 1. (B) Effects of various amounts of G2M-phase cytotoxic molecules perfused in network 1 once a week, by varying the duration of the perfusion $d$.

Protocols A and B produced the same effects. In the next simulations (Fig. 11B) we further reduced the amount of drug delivered so as to determine the smallest amount necessary to eliminate the tumour. As in protocol A and B, the frequency for drug administration is fixed to one week. By reducing the dose delivered, a decreasing amount of tumour cells is killed following each perfusion. The population of tumour cells falls below the detection threshold in 21 days for a perfusion duration down to 4 hours. If we keep lowering the amount of drug delivered at each cycle down to a factor 2, the tumour can still be eliminated in 28 days (that is with just one more cycle of perfusion). The optimum dose thus corresponds to a perfusion of 2 hours every week since a smaller dose is not sufficient to prevent the tumour from growing. 


\subsection{Vascular Disrupting Agents}

\subsubsection{Experimental observations}

Two-photon microscopic images of the tumor vasculature in a human lung carcinoma HTB177 model, growing in a dorsal skin fold chamber at the back of a nude mouse, have been acquired to observe the effects of VDAs. A fluorescent blood agent was injected intravenously for the staining of the tumor vasculature (Fig. 12A) [22]. Thirty minutes after intraperitoneal injection of the vascular disrupting agent, an increase of the vascular permeability was observed and the blood pool agent diffused across vascular endothelium (Fig. 12B). This effect is exhibited in the tumour region with figures $12 \mathrm{C}$ and 12D. Shortly afterwards, blood flow in tumor vessels stopped whereas in vessels at the tumour periphery, red blood cells were still circulating (Fig. 12E). One hour after VDA treatment, a second injection of a blood agent was performed and nearly no perfusion was observed in the tumor area (Fig. 12F).

\subsubsection{Numerical simulations}

A five seconds bolus injection of VDA is administered. Figure 13 shows the effects of the treatment, simulated from the computational model, where the vasculature, oxygen field and tumour state are compared in figures 13(A,D), 13(B,E), 13(C,F) respectively. Some vessels are disrupted (according to the rules defined in section 2.3.2.) inducing blood flow shutdown (Fig. 13D) associated to the occurence of large hypoxic areas in the tumour mass (Fig.13E). The tumour cells located in these areas rapidly become necrotic and the tumour is substancially but also only partially affected (Fig. 13F). This simulation result shows that the injection of VDAs can reduce the size and growth rate of the tumour. However, the tumour cannot be eradicated with VDAs alone in that case since the remaining tumour mass, rich with proliferative cells, will obviously regrow if treatment is stopped.

Figure 14B shows the evolution of the different states of the tumour cells population over 15 days after the injection of VDAs and compares it to the case without treatment (Fig. 14A). The effects of the VDAs are instantaneous, but combined with the loss of proliferative cells due to the "artificial" initial condition where the tumour spontaneously adapts to the vasculature. The adaptation mechanism is shown, in the case without treatment, by the drop in proliferative cells $(\mathrm{P})$ which become necrotic (N) (Fig. 14A). The tumour then stabilizes in about 2 days. But this stable state only lasts for ten days corresponding to the time limit beyond which the quiescent tumour cells eventually die through necrosis according to our choice for this parameter.

The injection of VDAs significantly amplifies the decrease of the population of proliferative cells $(\mathrm{P})$ which either become quiescent $(\mathrm{Q})$ or necrotic $(\mathrm{N})$ depending on the degree of hypoxia they are experiencing. The apoptotic cells are not explicitly represented in this graph, however the difference between the tumour cells alive $(\mathrm{P}+\mathrm{Q})$ and dead $(\mathrm{N})$ gives the rate of apoptotic death. Measures realised 15 days after treatment reveal a loss of about $20 \%$ of proliferative cells and an increase of about $40 \%$ of necrotic cells compared to the case without treatment. Regrowth of the 
vascular network is not taken into account in this model, however it is expected that this would allow some quiescent cells to switch back to proliferation. The tumour will ultimately regrow if nothing is undertaken to prevent it.

\section{Combination with cytotoxic molecules}

The aim with the use of VDAs is to cut the blood delivery to the tumour cells so as to starve them to death. What is typically observed with VDAs is massive tumour necrosis as in our simulation of figure 13. Surviving cells can however allow the tumour to regrow. This is why VDAs should preferentially be used in combination with other therapies [33,5]. The combination of the actions of VDAs with cytotoxic molecules is now envisaged. Figure 15 presents two cases, where VDAs are used before (Fig. 15A) or after (Fig. 15B) cytotoxic perfusion cycles. A lower dose of cytotoxic drug is considered here with a 3 hour perfusion rather than 5 . In both cases, an initial drop of $65 \%$ of the amount of proliferative cells is observed. Tumour regrowth is slightly reduced when the vascular network is initially altered with VDAs (Fig.15A). However, these alterations are not sufficient to kill the tumour through hypoxia nore to improve the cytotoxic delivery to all tumour cells, since the remaining clusters of proliferative cells constantly regrow. In the other hand, if VDAs are used once a significant reduction of the amount of proliferative cells has been reached then elimination of the remaining proliferating cells can be obtained. The use of VDAs in that case, allows one to spare the patient more cycles of cytotoxic perfusion.

\section{Conclusion}

This preliminary work aimed at showing that is it possible to get insights, through numerical simulations, into the complexity of the tumour evolution once a therapy is applied. Our aim at this stage was therefore purely illustrative with no quantitative ambition such as the definition of optimum dosage.

We have first tested two different phase-specific cytotoxic molecules. Unsurprisingly, the molecule acting on the longest phase is more efficient since the probability for the cells to be in the right phase, when treatment is applied, is higher. Moreover we have noted that the density of the vascular network perfusing the tumour enhance the uptake of drug by the tumour, however this enhanced perfusion also favours cells proliferation and tumour regrowth between two cycles of drug delivery. Since unperfused tissue zones lead to necrosis, there is consequently no qualitative difference between a highly perfuse tumour and a less perfused one concerning cytotoxic effects. Variations of the parameters defining the therapeutic protocol, that is the duration of the perfusion (directly related to the amount of drug delivered) and the periodicity of the perfusion cycles allows one to chose the best options based on these results. For example our simulations permit to define the most efficient protocol which is the one using the smallest amount of drug or alternatively the one that would allow the patient a longer period of recovery between sessions of drug administration in case of potential severe side-effects induced by the treatment. 
Effects of VDAs were also tested. We started by illustrating the effects of VDAs from twophoton microscopy experiments performed on a mouse bearing a tumour in a dorsal skinfold chamber. VDAs administration led to enhance vascular permeability immediately followed by a shutdown in blood flow for some vessels perfusing the tumour. Our simulation could reproduce this effect by targetting the low shear rate vessels, that is where the VDAs are given the highest probability to act. Shutdown of flow led to hypoxia resulting in massive tumour necrosis. Although the impact on the tumour was significant, simulation shows that it is not enough to eradicate all proliferative tumour cells. Combination with cytotoxic molecules is one efficient mean to reach this goal. Post treatment with VDAs allows one to isolate the remaining clusters of proliferative cells which eventually die of hypoxia.

We finally wish to draw attention on our choice of parameters used for the simulations. Those have been arbitrarily chosen since our aim was not directed towards quantitative predicitions. The parameters are consequently not specific of a particular tumour type, they can however be adjusted in that sense. It can be argued that a 3 days long cell cycle is an over-estimation of the duration of tumour cells cycles, however this parameter remains difficult to estimate in vivo. The time during which a cell can remain quiescent that we set to 10 days is in the other hand probably underestimated since some cells can remain dormant for several months.

At this stage, our aim was to propose a computational framework to get insights into the outcome of potential cytotoxic and VDAs treatment and this goal was attained. In a next stage, our aim will be to test further this framework for a specific tumour type and to validate the model prediction from our in vivo experiment on mice bearing tumours in dorsal skinfold chambers.

The validation of such a computational tool would allow to optimize the therapy while limiting side effects for the patients. Application of such a tool in the clinic would help to rationalize the use of existing therapeutic modes by guiding the clinician to decide on the best possible strategy (drug combination, dose, protocol). Simulations can now be performed on standard multicore workstations with a reasonable timescale ranging from a few minutes, for assessing the effect of a given drug, to the hour to find the optimum treatment among all the possibilities. This opens up new perspectives towards Computer-Assisted Therapeutic Strategies.

\section{Acknowledgements}

This work was funded by the French National Network and Rhône-Alpes Institute for Complex Systems (RNCS and IXXI). We acknowledge Cyril Zenga (Ecrins Therapeutics) for biotechnical assistance and François Estève (Grenoble Institute of Neurosciences) for his support in the development of this project. 


\section{References}

[1] T. Alarcon, H. Byrne, P.K. Maini. A cellular automaton model for tumour growth in inhomogeneous environment. J. Theor. Biol., 225 (2003), No. 2, 257-74.

[2] T. Alarcon, H. Byrne, P.K. Maini. A mathematical model of the effects of hypoxia on the cell-cycle of normal and cancer cells. J. Theor. Biol., 229 (2004), No. 3, 395-411.

[3] A. Altinok, D. Gonze, F. Lévi, A. Goldbeter. An automaton model for the cell cycle. Interface Focus, 1 (2011), 36-47.

[4] A.R.A. Anderson, K.A. Rejniak, P. Gerlee, V. Quaranta. Modelling of cancer growth, evolution and invasion: bridging scales and models. Math. Mod. Nat. Phenom., 2 (2007), No. 3, $1-29$.

[5] B.C. Baguley, D.W. Siemann. Temporal aspects of the action of ASA404 (vadimezan; $D M X A A)$. Expert Opin. Investig. Drugs., 19 (2010), No. 11, 1413-25.

[6] H.M. Byrne. Dissecting cancer through mathematics: from cell to the animal model. Nat. Rev. Cancer, 10 (2010), 221-30.

[7] P. Carmeliet. Angiogenesis in life, disease and medicine. Nature, 438 (2005), No. 7070, 9326.

[8] J.J. Casciari, S.V. Sotirchos, R.M. Sutherland. Variations in tumor cell growth rates and metabolism with oxygen concentration, glucose concentration, and extracellular PH. J. Cell Physiol., 151 (1992), No. 2, 386-94.

[9] A. d'Onofrio, A. Gandolfi. Chemotherapy of vascularised tumours: role of vessel density and the effect of vascular "pruning". J. Theor. Biol., 264 (2010), 253-65.

[10] A. Eichholz, S. Merchant, A.M. Gaya. Anti-angiogenesis therapies: their potential in cancer management. OncoTragets and Therapy, 3 (2010), 69-82.

[11] J. Folkman. Tumor angiogenesis: therapeutic implications. N. Engl. J. Med., 285 (1971), No. 21, 1182-6.

[12] J.P. Freyer, E. Tustanoff, A.J. Franko, R.M. Sutherland. In situ oxygen consumption rates of cells in V-79 multicellular spheroids during growth. J. Cell Physiol., 118 (1984), 53-61.

[13] J.P. Freyer, R.M. Sutherland. Regulation of growth saturation and development of necrosis in EMT6/Ro multicellular spheroids by the glucose and oxygen supply. Cancer Res., 46 (1986), 3504-3512.

[14] J.L. Gevertz. Computational modeling of tumor response to vascular-targeting therapies part I: validation. Comput. Math. Methods Med., (2011), 830515. 
[15] J. Grote, R. Süsskind, P. Vaupel. Oxygen diffusivity in tumor tissue (DS-carcinosarcoma) under temperature conditions within the range of 20-40 degrees C. Pflugers Arch., 372 (1977), No. 1, 37-42.

[16] C.A. Honstvet. Targeting tumour vasculature as a cancer treatment. Comp. Math. Meth. Med., 8 (2007), No. 1, 1-9.

[17] T. Hoshino, C.B. Wilson, M.L. Rosenblum, M.J. Barker. Chemotherapeutic implications of growth fraction and cell cycle time in glioblastomas. Neurosurg., 43 (1975), 127-35.

[18] R.K. Jain. Normalizing tumor vasculature with anti-angiogenic therapy: a new paradigm for combination therapy. Nat. Med., 7 (2001), No. 9, 987-9.

[19] J.W. Lippert. Vascular disrupting agents. Bioorg. Med. Chem., 15 (2007), No. 2, 605-15.

[20] J.S. Lowengrub, H.B. Frieboes, F. Jin, Y.L. Chuang, X. Li, P. Macklin, S.M. Wise, V. Cristini. Nonlinear modelling of cancer: bridging the gap between cells and tumours. Nonlinearity, 23 (2010), R1-R91.

[21] P. Macklin, S.R. McDougall, A.R.A. Anderson, M.A.J. Chaplain, V. Cristini, J. Lowengrub. Multiscale modelling and nonlinear simulation of vascular tumour growth. J. Math. Biol., 58 (2009), No. 4-5, 765-98.

[22] M. Maurin, O. Stéphan, J.C. Vial, S.R. Marder, B. van der Sanden. Deep in vivo Two-Photon Imaging of Blood Vessels with a new Dye encapsulated in Pluronic Nanomicelles. J. Biomed. Opt., 16 (2011), 036001.

[23] S.R. McDougall, A.R.A. Anderson, M.A.J. Chaplain. Mathematical modelling of dynamic adaptative tumour-induced angiogenesis: clinical implications and therapeutic targeting strategies. J. Theor. Biol., 241 (2006), 564-89.

[24] S.R. McDougall, M.A.J. Chaplain, A. Stéphanou, A.R.A Anderson. Modelling the impact of pericyte migration and coverage of vessels on the efficacy of vascular disrupting agents. Math. Mod. Nat. Phenom., 5 (2010), No. 1, 163-202.

[25] T. Morimura. Prolongation of G1 phase in cultured glioma cells by cisdichlorodiammineplatinum (II) (CDDP): Analysis using bromodeoxyuridine (BrdU)-Hoechst technique. J. Neuro-Oncol., 7 (1989), 71-79.

[26] L.J. Nugent, R.K. Jain. Extravascular diffusion in normal and neoplastic tissues. Cancer Res., 44 (1984), No. 1, 238-44.

[27] J.M. Osborne, A. Walter, S.K. Kershaw, G.R. Mirams, A.G. Fletcher, P. Pathmanathan, D. Gavaghan, O.E. Jensen, P.K. Maini, H.M. Byrne. A hybrid approach to multi-scale modelling of cancer. Philos. Transact. A Math. Phys. Eng., 368 (2010), No. 1930, 5013-28. 
[28] M.R. Owen, I.J. Stamper, M. Muthana, G.W. Richardson, J. Dobson, C.E. Lewis, H.M. Byrne Mathematical modeling predicts synergistic antitumour effects of comibining a macrophagebased, hypoxia-targeted gene therapy with chemotherapy. Cancer. Res., 71 (2011), No. 8 , 2826-37.

[29] M. Pàez-Ribes, E. Allen, J. Hudock, T. Takeda, H. Okuyama, F. Viñals, M. Inoue, G. Bergers, D. Hanahan, O. Casanovas. Antiangogenic therapy elicits malignant progression of tumors to increased local invasion and distant metastasis. Cancer Cell., 15 (2009), No. 3, 220-31.

[30] J. Panovska, H.M. Byrne, P.K. Maini. A theoretical study of the response of vascular tumours to different types of chemotherapy. Math. Comp. Mod., 47 (2008), 560-79.

[31] H. Perfahl, H.M. Byrne, T. Chen, V. Estrella, T. Alarcon, A. Lapin, R.A. Gatenby, R.J. Gillies, M.C. Lloyd, P.K. Maini, M. Reuss, M.R. Owen. Multiscale modelling of vascular tumour growth in 3D: the roles of the domain size and boundary conditions. PLoS ONE, 6 (2011), No. 4, e14790.

[32] B. Pertuiset, D. Dougherty, C. Cromeyer, T. Hoshino, M. Berger, M.L.J Rosenblum. Stem cell studies of human malignant brain tumours. Part $2:$ proliferation kinetics of brain-tumour cells in vitro in early-passage cultures. Neurosurg., 63 (1985), 426-32.

[33] F. Rehman, G. Rustin. ASA404: update on drug development. Expert Opin. Investig. Drugs, 17 (2008), No.10, 1547-1551.

[34] R. Rockne, J.K. Rockhill, M. Mrugula, A.M. Spence, I. Kalet, K. Hendrickson, A. Lai, T. Cloughesy, E.C. Alvord Jr, K.R. Swanson. Predicting the efficacy of radiotherapy in individual glioblastoma patients in vivo: a mathematical modeling approach. Phys. Med. Biol., 55 (2010), 3271-85.

[35] R.J. Shipley, S.J. Chapman. Multiscale modelling of fluid and drug transport in vascular tumours. Bull. Math. Biol., 72 (2010), No.6, 1464-91.

[36] D.W. Siemann, E. Mercer, S. Lepler, A.M. Rojiani. Vascular targeting agents enhance chemotherapeutic agent activities in solid tumor therapy. Int. J. Cancer, 99 (2002), 1-6.

[37] D.W. Siemann, M.R. Horsman. Enhancement of radiation therapy by vascular targeting agents. Curr. Opin. Investig. Drugs, 3 (2002), 1660-5.

[38] D.W. Siemann, M.R. Horsman. Vascular targeted therapies in oncology. Cell Tissue Res. 335 (2009), No.1, 241-248.

[39] G.S. Stamatakos, V.P. Antipas, N.K. Uzunoglu, R.G. Dale. A four-dimensional computer simulation model of the in vivo response to radiotherapy of glioblastoma multiforme: studies on the effect of clonogenic cell density. The British Journal of Radiology, 79 (2006), 389-400. 
[40] A. Stéphanou, S.R. McDougall, A.R.A Anderson, M.A.J. Chaplain. Mathematical modelling of flow in $2 d$ and $3 d$ vascular networks: applications to anti-angiogenic and chemotherapeutic drug strategies. Math. Comp. Mod., 41 (2005), No. 10, 1137-56.

[41] A. Stéphanou, S.R. McDougall, A.R.A Anderson, M.A.J. Chaplain. Mathematical modelling of the influence of blood rheological properties upon adaptative tumour-induced angiogenesis. Math. Comp. Mod., 44 (2006), No. 1-2, 96-123.

[42] E.A. Swabb, J. Wei, P.M. Gullino. Diffusion and convection in normal and neoplastic tissues. Cancer Res., 34 (1974), No. 10, 2814-22.

[43] K.R. Swanson, E.C. Alvord, J.D. Murray. Quantifying efficacy of chemotherapy of brain tumours with homogeneous and heterogeneous drug delivery. Acta. Biotheor., 50 (2002), No. 4, 223-37.

[44] G. Tanaka, Y. Hirata, S.L. Goldenberg, N. Bruchovsky, K. Aihara. Mathematical modelling of prostate cancer growth and its application to hormone therapy. Phil. Trans. R. Soc. A, 368 (2010), 5029-44.

[45] G.M. Tozer, C. Kanthou, B.C. Baguley. Disrupting tumour blood vessels. Nat. rev. Cancer, 5 (2005), No. 6, 423-35.

[46] P. Tracqui. Biophysical models of tumour growth. Rep. Prog. Phys., 72 (2009), No. 5, 056701.

[47] J.T. Tyson, B. Novak. Temporal organization of the cell cycle. Curr. Biol., 18 (2008), No. 17, R759-R768.

[48] B. Wang, J.M. Rosano, R. Cheheltani, M.P. Achary, M.F. Kiani. Towards a targeted multidrug delivery approach to improve therapeutic efficacy in breast cancer. Expert Opin. Drug Deliv., 7 (2010), No. 10, 1159-73. 


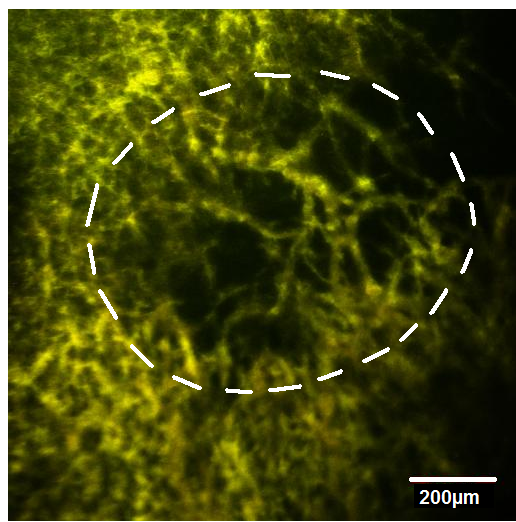

(A)

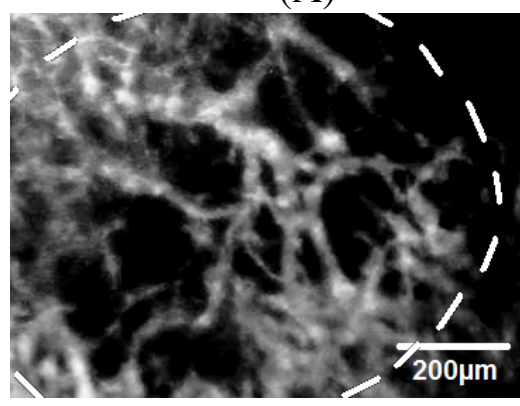

(C)

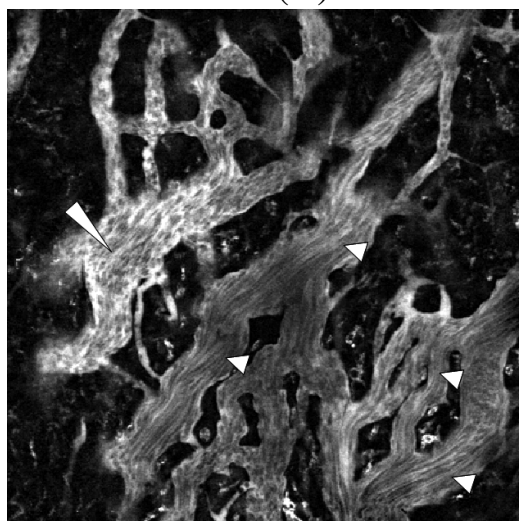

(E)

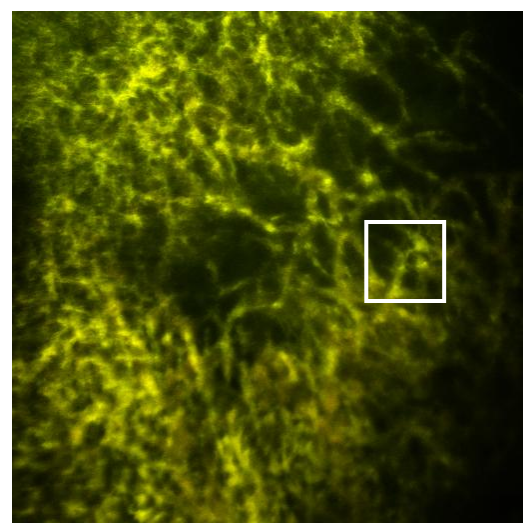

(B)

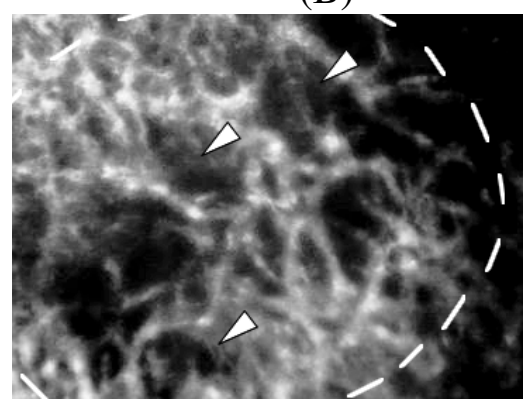

(D)

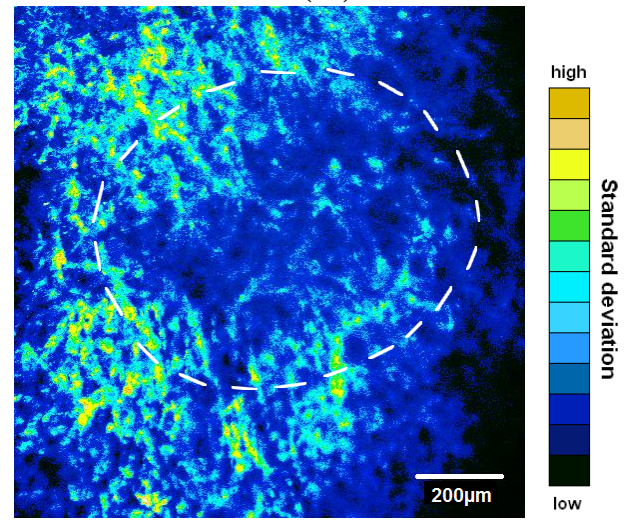

(F)

Figure 12: Effects of the VDAs from two-photon microscopic images. (A) Vascular network before treatment. The tumour area is indicated with the dashed line. (B) Vascular network 30 minutes after VDAs injection. The higher fluorescence intensity of the backgroung reveals vascular permeability induced by VDAs. (C) and (D) are extracted from (A) and (B) respectively to exhibit the effect of the VDAs in the tumour area. The arrows in figure (D) show the main spots where the blood leaked out of the vessels. (E) Detail of (B) where red blood cells are seen as dark spots in the blood plasma where the flow is stopped (long arrow) whereas circulating blood cells are seen as dark stripes inside the perfused vessels (small arrows). Images (A,B) are Z-projections (average intensities) of Z-stacks of 33 slices with interdistances of 5 microns. In (F) a Z-projection of standard deviations of fluorescence intensities show only regions with blood perfusion around the tumor area characterized by high standard deviations. 


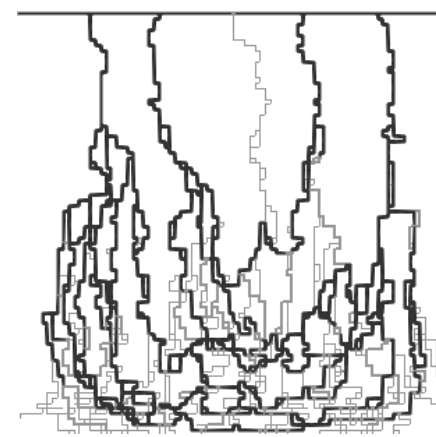

(A)

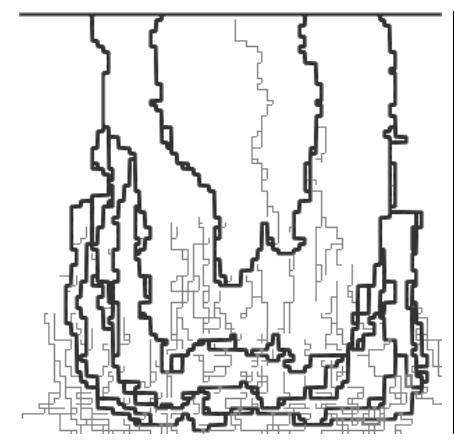

(D)

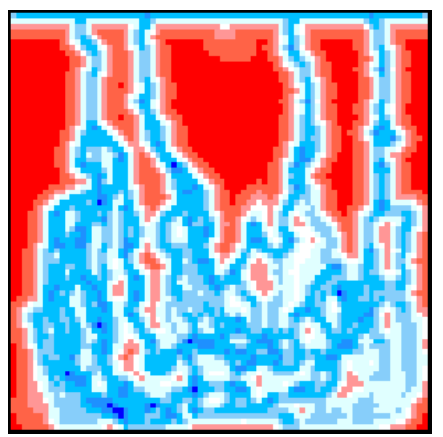

(B)

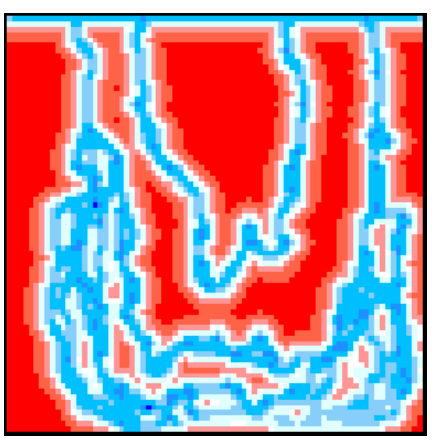

(E)

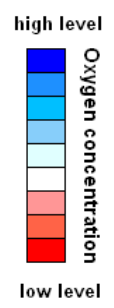

\begin{tabular}{c} 
high level \\
\hline$\square$ \\
\hline \\
\hline \\
\hline \\
\hline
\end{tabular}

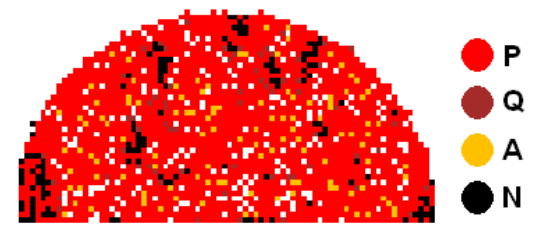

(C)

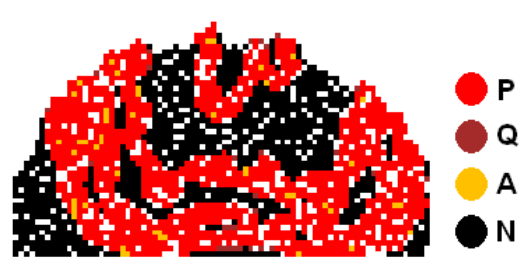

(F)

Figure 13: Simulation of the effect of the VDA. (A,B,C) before treatment and $(\mathrm{D}, \mathrm{E}, \mathrm{F})$ after treatment. (A,D) vascular network; $(\mathrm{B}, \mathrm{E})$ oxygen concentration; $(\mathrm{C}, \mathrm{F})$ tumour mass.

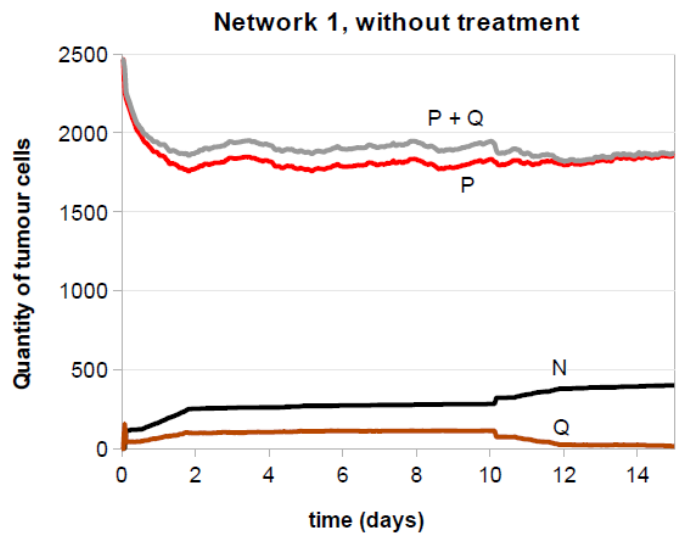

(A) Without treatment

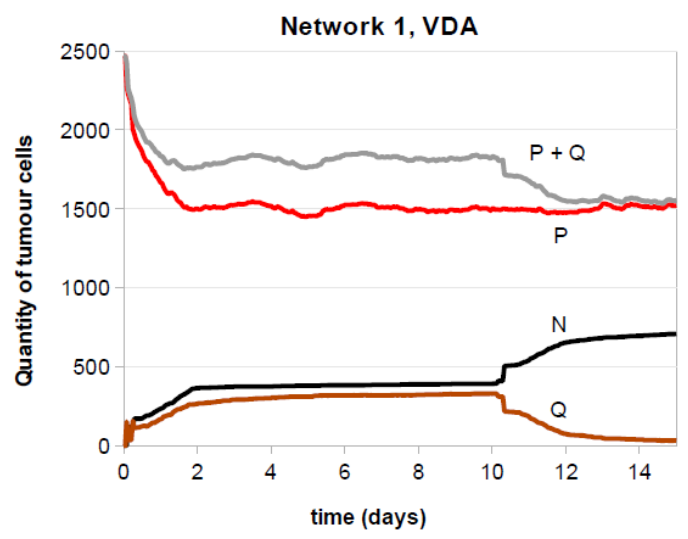

(B) With VDA treatment

Figure 14: Comparison of the evolutions of the populations of tumour cells including proliferative $(\mathrm{P})$, quiescent cells $(\mathrm{Q})$ and necrotic cells $(\mathrm{N})$ without treatment $(\mathrm{A})$ and after injection of VDAs (B) in vascular network 1 . 


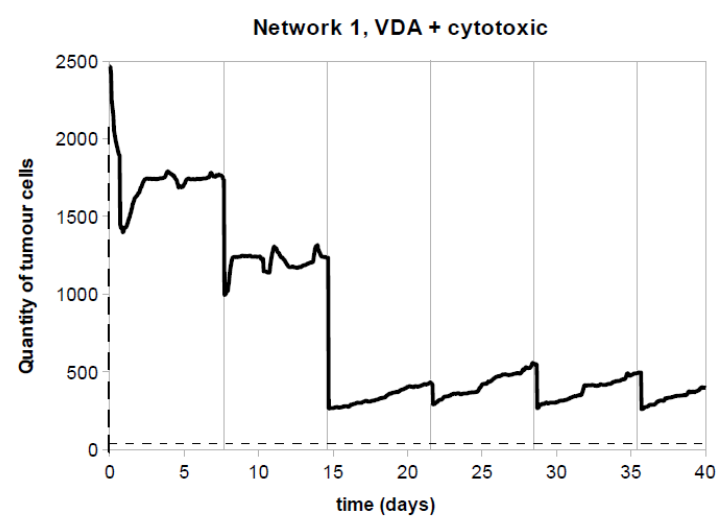

(A) VDA + cytotoxic

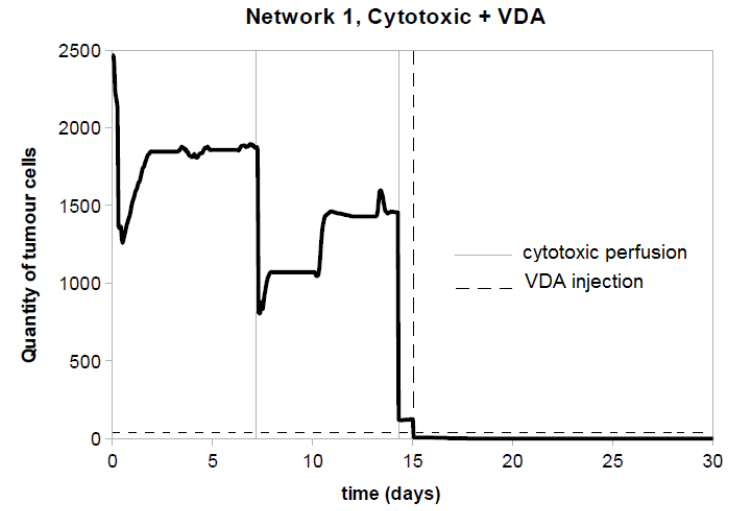

(B) cytotoxic + VDA

Figure 15: Effects of the combined used of cytotoxic molecules and VDAs applied to the tumour perfused with vascular network 1 . 


\section{Appendix}

\section{A Coupling tumour growth and flow time scales}

A large discrepancy exists between the time scales for cell proliferation $\left(10^{2} h\right)$, oxygen diffusion (1 to $10 s)$, cytotoxic drug injection $\left(10\right.$ to $\left.10^{2} h\right)$ and diffusion (1 to $\left.10 s\right)$ and VDA injection (1 to $10 s$ ) and diffusion (1 to $10 s)$.

In order to manage this multiple time scales, two different time scales are used to handle fast and slow processes $\left(\tau \approx 10^{-4} s\right.$ and $\left.\hat{\tau} \approx 10 h\right)$. The procedure used to couple these two scales is as follows. When a drug is administered, its injection and diffusion is modeled using the fast time scale $\tau$ and tumour evolution is paused. Time scales are switched to the slow scale $\hat{\tau}$, in order to describe the tumour evolution, as soon as one of the following conditions happens depending on the injected drug :

- if the drug is a cytotoxic drug: when the drug reaches a steady state,

- if the drug is a VDA: when no more vessels are disrupted, vasculature adaptation is done and oxygen reaches a steady state. 